\title{
Global cooperativity of the short-range process in apparent movement: Evidence obtained with contour-containing stimuli
}

\author{
J. TIMOTHY PETERSIK \\ Ripon College, Ripon, Wisconsin
}

\begin{abstract}
Previous research has demonstrated that the short-range process in apparent movement, as studied with random-dot cinematograms, exhibits global cooperativity; that is, computations performed by local elements interact nonlinearly and are pooled. Other research using displays containing extended contours has implicated the short-range process, but has never demonstrated global cooperativity. In the first of four experiments, it was shown that under certain conditions of presentation, a short-range motion percept exhibiting apparent global cooperativity can be obtained when collections of randomly located contours are rotated about the center of a display, despite the fact that the displacement of peripheral contours falls outside the normal limit of the short-range process. Experiments 2-4 were conducted to provide further evidence that the observed motion is short-range (i.e., it can be disrupted by illuminating the interstimulus interval or with dichoptic viewing) and that the percept is globally cooperative (i.e., masking the center of the display, where separations between corresponding elements across frames are smallest, results in a decline in the frequency of reports of the short-range percept). Control observations suggest that the effect produced with masks was not due to a decrease in the number of elements in the display. The argument that the display exhibits a short-range process with global cooperativity is further developed.
\end{abstract}

A perceptual process can be said to exhibit cooperativity when spatially adjacent local elements of the process interact nonlinearly with one another (Chang \& Julesz, 1985). The process is said to be global if the computations performed by the local elements are pooled and result in a coherent percept. Julesz (1971) showed how a global cooperative process could account for the percept of a figure in depth that results when random-dot frames containing correlated and displaced regions are viewed stereoscopically. Chang and Julesz $(1984,1985)$ showed that the figural percept resulting when similar randomdot frames are alternated in apparent movement (AM) can also be accounted for by a global cooperative process. Since the AM process underlying the segregation of correlated, displaced regions in alternating random-dot frames is the "short-range" process (so called because, among its properties, it is limited to a relatively short spatial integration range; Braddick, 1974), the inference from Chang and Julesz's experiments is that the short-range process is global and cooperative.

I am grateful to Cathy Guarnieri, Beth Laufenberg, Amy Rosner, and Sherry Schwandt for their competent assistance in data collection, their suggestions regarding experimental procedure, and their comments regarding data interpretation. I also thank Norm Loomer for assisting with statistical decisions, Mike Braunstein for patiently encouraging and helping me to consider a correspondence-matching explanation of the data, and Bonnie Wolff for her careful preparation of the manuscript. Correspondence may be addressed to J. Timothy Petersik, Department of Psychology, Ripon College, P.O. Box 248, Ripon, WI 54971.
Current literature on apparent motion has shown that the short-range process can be revealed in two types of displays. One of these is the random-dot ( $r-d)$ displays studied by Braddick and by Chang and Julesz. Formally, $r-d$ displays consist of large arrays (typically on the order of $100 \times 100$ ) of black-and-white pixels. When viewed individually, the frames of such displays show no global contours or forms. However, if a given area of random dots is displaced a short distance between frames and reproduced in both, alternation of the frames can result in the appearance of a "figure" that stands out from its background. In such $r-d$ displays, the globally cooperative short-range process is responsible for the generation of the perceived form. The other type of display used to study the short-range AM process is based on sets of larger contours. For example, Petersik and Pantle (1979) studied a display consisting of a row of three solid dots (about $0.5^{\circ}$ diam) that were uniformally displaced horizontally by their interdot distance from frame to frame. Similarly, Gerbino (1984) studied short-range phenomena using a display consisting of triangles whose horizontal and vertical dimensions were about $4.5^{\circ}$ and $2^{\circ}$, respectively. These "form-containing" frames yielded bistable percepts: at short interstimulus intervals (ISIs), some shortrange percept is visible, and at long ISIs a long-range percept is seen (see Petersik, 1989, for a review). These displays also differ from the $r-d$ displays in that the shortrange process does not generate new forms; rather, its perceptual manifestation is solely of AM over small distances or stationarity (e.g., Pantle \& Petersik, 1980). 
Despite a rather sizable body of literature on the shortrange process (Petersik, 1989), its global cooperativity has been demonstrated only with $\mathrm{r}-\mathrm{d}$ displays. To date, no demonstration of the global cooperativity of the shortrange process has appeared with displays consisting of frames that contain large contours or forms. It is desirable to determine whether the short-range motion observed with the alternation of form-containing frames exhibits global cooperativity because, although the AM processes underlying the generation of figures in $\mathrm{r}-\mathrm{d}$ displays and the local motion percepts observed with bistable displays have both been called "short-range" (because their spatiotemporal constraints are similar), there has been no independent evidence to demonstrate that the same underlying process is responsible for both phenomena. Evidence that the short-range percepts obtained with the alternation of form-containing frames result from a global cooperative process would support the argument that the same short-range AM process operates in both cases.

In the present paper, a display that consists of frames which contain contoured forms and which shows shortrange $A M$ phenomena is described. It is argued that one of the two percepts seen with this display exhibits global cooperativity. The display itself is a variation of ones used previously by Bell and Lappin (1979) and by Bischof and Groner (1985) in their studies of short- and long-range AM phenomena. However, these previous authors did not consider the question of global cooperativity. Briefly, the present display consists of a set of 40 dots randomly placed within the invisible confines of a larger circle (see Figure 1). ${ }^{1}$ From frame to frame of the AM display, this circle is rotated about its center by some specified amount.

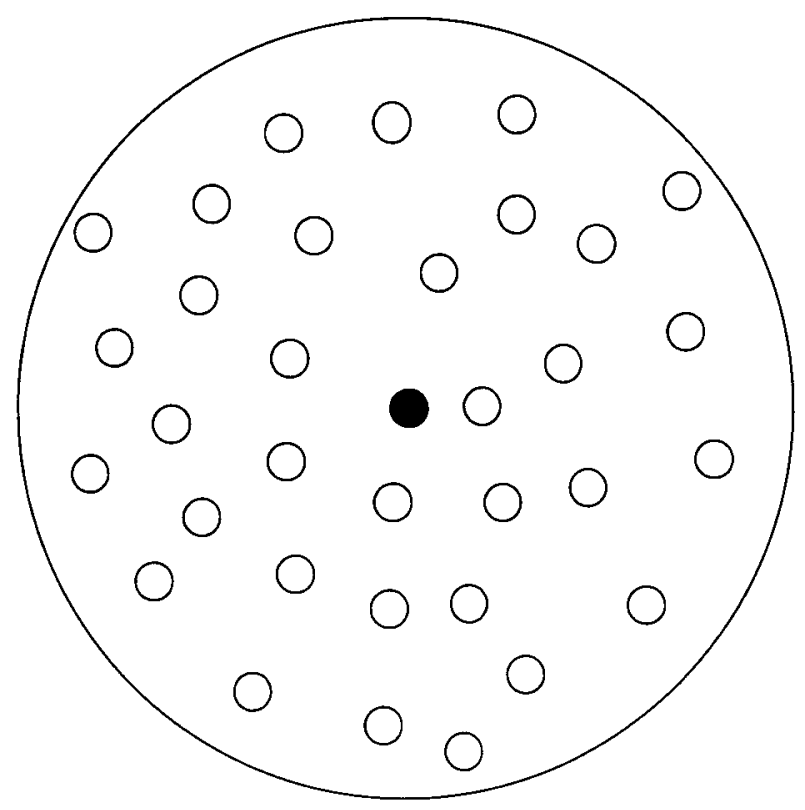

Figure 1. An example of the kind of stimulus used in the present experiments. The perimeter of the larger circle was not visible in the experiments. Also, the number of open stimulus dots was varied.
If the ISI is relatively short and the degree of rotation not too large (the two factors interact), subjects report a global back-and-forth rocking percept. However, if the ISI is made too long or the rotation too large, subjects report a random, incoherent motion of the individual dots of the display. As will be shown below, the rocking percept is thought to result from the influence of the short-range process because its temporal and spatial limits correspond well to those exhibited with other short-range AM displays (e.g., Gerbino, 1984; Petersik \& Pantle, 1979). Although the incoherent motion has not been studied extensively, it is possible that it results in part from unpooled (i.e., nonglobal, noncooperative) actions of the shortrange process and in part from the long-range process.

\section{EXPERIMENT 1}

\section{Method}

Stimuli and Apparatus. The stimuli were prepared in the following way. First, a template was prepared by cutting a circle out of a piece of graph paper. Forty cells of the circle were randomly chosen and holes were made at their locations by a hole puncher, subject to the following constraints: (1) each quadrant of the circle had $10 \pm 1$ holes, and (2) holes were no closer than $0.5 \mathrm{~cm}\left(11^{\prime}\right)$ edge to edge. The template was then placed over the center of a stimulus card $(17.5 \times 12.5 \mathrm{~cm})$ and rotated by a specified amount if necessary. Next, the circumference of each dot on the template was traced in india ink. When viewed from a distance of $153 \mathrm{~cm}$, the outer-edge-to-outer-edge diameter of each dot subtended $0.22^{\circ}$ (or about 13.5'). The line demarcating the circumference of the dot subtended 2.3'. The diameter of the (invisible) circle in which the dots lay subtended $4.2^{\circ}$. On the center of each card was drawn a solid black dot of the same size as the other stimulus dots. This served as a fixation point during the experiment. When completed, there were six sets of stimulus cards, one for each of six angular rotations between the stimuli of each set. The angular rotations were $5^{\circ}, 10^{\circ}, 20^{\circ}, 40^{\circ}, 80^{\circ}$, and $135^{\circ}$.

Each of the two frames of a display was presented through one channel of a three-channel tachistoscope (Scientific Prototype, Model GB). Stimuli were drawn in india ink on white cards, and these subtended a visual angle of $6.6^{\circ}$ horizontally and $4.8^{\circ}$ vertically. The luminance of the white parts of the stimuli was $4.2 \mathrm{~cd} / \mathrm{m}^{2}$; of the black, $0.67 \mathrm{~cd} / \mathrm{m}^{2}$.

Procedure. Subjects were run individually. Upon arrival, each subject was told the nature of the task and was shown two diagrams that depicted the types of motion that could be perceived during the experiment. One type of motion was called "rocking" motion because all of the dots of the display could be seen to pivot back and forth around the fixation point. The other type of motion was referred to as "random" motion because each dot could be perceived to move in some direction independent of the others. For the purpose of establishing response criteria, the subjects were instructed to identify only the displays in which all dots participated in global pivotting as "rocking." Displays with any noticeable deviations from global rocking were to be called "random." The subjects were told that if they perceived some other type of motion during the experiment they were to describe it carefully. This occurred rarely, and when it did the description was recorded and the trial was rerun later in the session. ${ }^{2}$

The subjects were next shown to a light-proof observation box in which eyepieces had been placed for observation of the tachistoscopic displays. They were then shown three examples of rocking motion and three of random motion as practice and as anchors for future judgments. The timing of the practice displays was different from any used during the experiment proper. 
The experiment consisted of the presentation of 24 displays that resulted from the factorial combination of the six rotation angles and four ISIs $(10,20,40$, and $80 \mathrm{msec})$. Each was viewed binocularly five times in each of two experimental sessions, making a total of 240 trials over two 60 -min sessions (120 trials/session). Trials were blocked in the following way. First, the stimuli corresponding to a single rotation angle were placed in the tachistoscope. Next, four trials corresponding to each of the four ISIs were run in a random order. A new rotation angle was then randomly selected, subject to the constraint that no rotation angle was repeated until each of the remaining five had been used once. During trials, the stimulus duration of each card was $200 \mathrm{msec}$ and the ISI was dark. Stimuli were shown for six cycles, where one cycle was Stimulus $1 \rightarrow$ ISI $\rightarrow$ Stimulus $2 \rightarrow$ ISI. Following the complete exposure of the stimuli, the subject was to respond either "rocking" or "random" as quickly as possible. No feedback was provided.

Subjects. The subjects were 18 volunteer psychology majors. Twelve of these were female, six male. Each reported normal vision, 9 requiring corrective lenses. All subjects were between the ages of 19 and 36 years.

\section{Results and Discussion}

Figure 2 shows the results of the experiment expressed as the percentage of "random" movement reported in each condition. A number of trends are apparent in the data. First, rotation angle was found to have a significant main effect in a repeated measures ANOVA $[F(5,85)=$ $114.79, p<.001]$ such that increases in rotation angle were associated with increases in reports of random movement. However, there were three groupings of data based on rotation angle. Data for $5^{\circ}$ and $10^{\circ}$ rotations were not significantly different, nor were data for $40^{\circ}, 80^{\circ}$, and $135^{\circ}$ rotations (both $p \mathrm{~s}>.05$ ). As can be seen in Figure 2, data points for the $40^{\circ}, 80^{\circ}$, and $135^{\circ}$ conditions overlapped substantially (top line of figure). The third group consisted of the data for the $20^{\circ}$ stimuli. Post hoc comparisons showed these three groups to be significantly different from one another (all $p s<.001$ ).
Figure 2 also shows an effect of ISI: as ISI increases, the percentage of random-movement reports increases for each grouping of data. Overall, this main effect was significant in the ANOVA $[F(3,51)=14.16, p<.001]$. A variety of curve-fitting routines were attempted with the three collections of data, and the best fits were found to be logarithmic. The best-fitting lines and their equations are shown in Figure 2 for the averages of the conditions contained in each grouping. $R^{2}$, or the proportion of variability in the data accounted for by the logarithmic relationship, is also shown for each grouping. In absolute terms, variations in ISI had greater effects on responses obtained with the $20^{\circ}$ stimuli than with the stimuli contained in the other two groups. Reasons for this difference will be considered below.

On the basis of the ISI effect, the abrupt changeover from a coherent to an incoherent percept at some critical ISI (a phenomenon visible in the data for individual subjects and discovered in pilot studies, but not readily apparent in the averaged data), and the subjective globality of the percept, it can be tentatively held that the rocking motion observed with the present displays is due to the short-range process in AM. This argument is supported by the finding of a significant main effect of rotation angle: as rotation increases, and thus as the distance between corresponding dots from frame to frame increases, the percentage of rocking percepts decreases (cf. Braddick, 1974; Pantle \& Petersik, 1980). However, the ANOVA also showed a significant rotation angle $\times$ ISI interaction $[F(15,255)=1.73, p<.05]$.

Figure 3 shows the growth of the linear distance between corresponding elements of the two frames of these displays as a function of their distance (on a radius) from the center of the invisible circle. Distances corresponding to four rotation angles are depicted: $5^{\circ}, 10^{\circ}, 20^{\circ}$, and $40^{\circ}$. This figure can be used to estimate the number

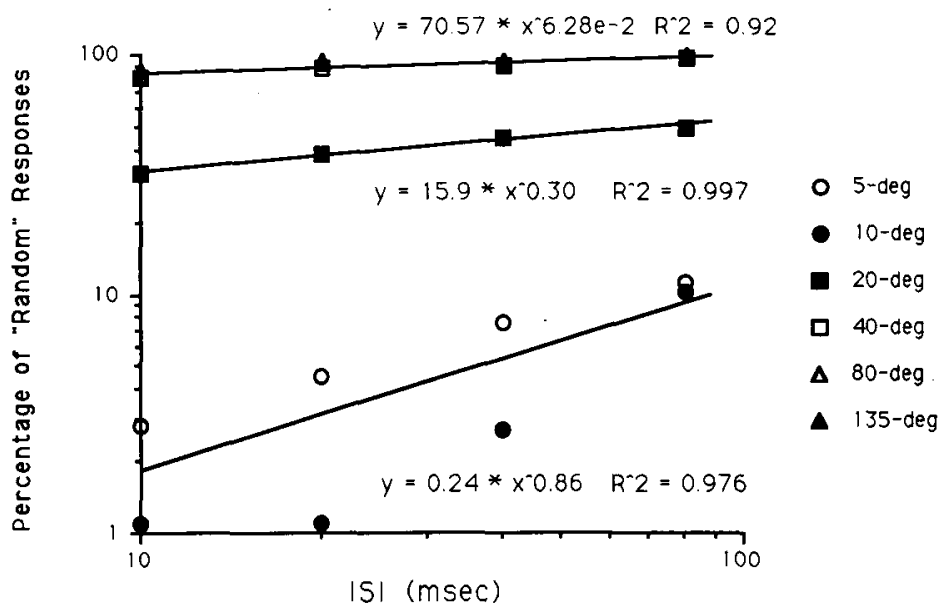

Figure 2. Results of Experiment 1. Because graphs in studies that have examined the short-range process with contour-containing stimuli have adopted the convention of showing the percentage of reports of the alternative percept (i.e., the non-shortrange percept) on the ordinate, here the percentage of "random" responses is shown as a function of ISI duration. The degree of rotation of corresponding elements across frames is the parameter. Best-fitting lines and logarithmic equations are shown for three salient collections of data. 


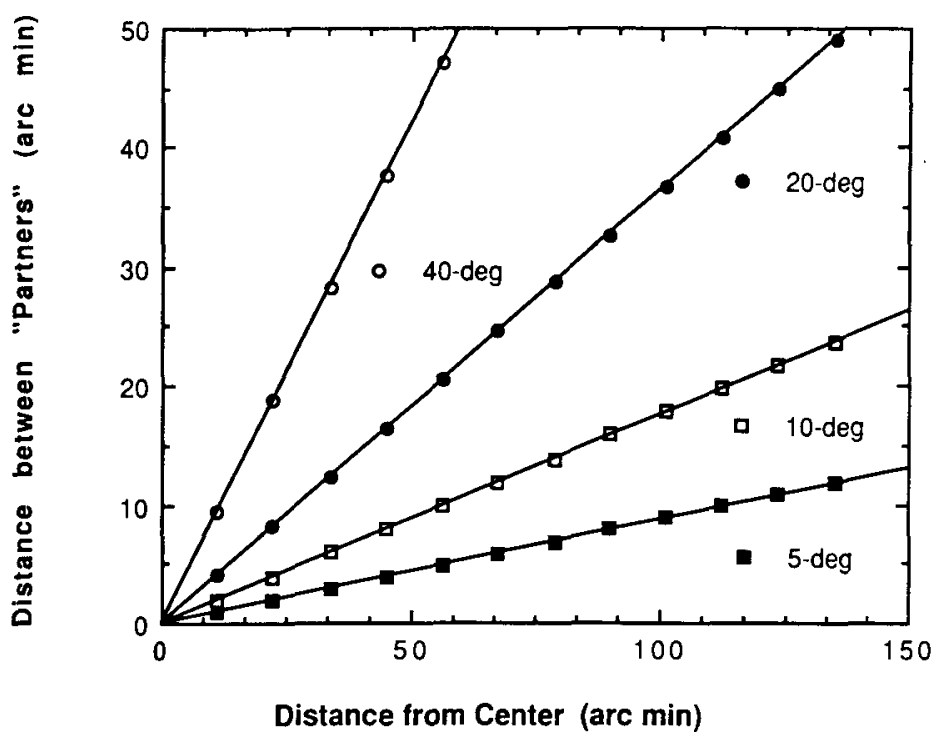

Figure 3. Growth of the linear distance between corresponding elements of the two frames of the AM display as a function of their distance from the display center. Each of the four lines shows this growth for a different magnitude of rotation between frames.

of points whose shifts between frames fall below and above a hypothetical spatial integration range of the shortrange process. For the present displays, that hypothetical range falls between a low of $15^{\prime}$ (as conservatively estimated by Braddick, 1974, to be an absolute limit of the short-range process, a point of view no longer well accepted; see Petersik, 1989) and a high of 16.6' (a liberal figure based on $123 \%$ of the dot diameter, as estimated by Petersik, Pufahl, \& Krasnoff, 1983). Using the $15^{\prime}$ figure only as a basis of comparison, it can be seen from Figure 3 that with a $5^{\circ}$ rotation, all dots (regardless of distance from center) are shifted within the putative spatial integration range of the short-range process. For the $10^{\circ}$ rotation stimuli, dots falling within about $80^{\prime}$ of the center lie within the short-range spatial limit; thus, about 25 dots lie within the spatial limit, about 15 outside it. The corresponding distances from the center that demarcate the hypothetical spatial limit of the short-range process for the $20^{\circ}$ and $40^{\circ}$ rotation stimuli are $60^{\prime}$ (about 19 dots inside and 21 dots outside) and 20' (about 6 dots inside and 34 dots outside). For rotations of $80^{\circ}$ and $135^{\circ}$, nearly all shifted dots fall outside the spatial integration range of the short-range process. On the basis of this analysis (along with the nonlinear effect of ISI), it is hypothesized that the short-range process exhibits global cooperativity in the present contour-containing displays: by definition, rocking motion was reported only when all dots of the display were seen in a synchronized rocking movement. Thus, for example, when rocking motion was seen with the $20^{\circ}$ rotation display, about 19 dots falling within the integration range of the short-range process influenced the perception of the 21 dots falling outside it.
The existence of some reports of rocking motion for stimuli rotated in the $40^{\circ}-135^{\circ}$ range requires additional explanation. For the $40^{\circ}$ rotation stimuli, it is possible that the approximately 6 dots falling within the hypothetical spatial integration range of the short-range process occasionally influenced the 34 dots lying outside that range sufficiently to produce a global percept of rocking movement. For the stimuli with larger rotations, it is possible that reports of rocking motion reflect a response bias on the part of the subjects. Alternatively, they may reflect guesses in situations in which the subjects were uncertain about the nature of their percepts. Under any circumstances, it is clear that reports of both rocking and random motion occur with some nonzero probability at any rotation.

If the hypothesis that in the general case rocking movement represents global cooperativity of the short-range process whereas random movement represents the breakdown of global cooperativity is true, this would explain the significant rotation angle $\times$ ISI interaction: ISI had a greater influence on the $20^{\circ}$ rotation condition than on the other two groupings of data. This may be due to the possibility that, spatially, most of the dots at the $5^{\circ}$ and $10^{\circ}$ rotations fall in or near the integration limit of the short-range process, whereas most of the dots at the $40^{\circ}-135^{\circ}$ rotations fall outside. Thus, ISI would have little opportunity to affect these percepts. However, at the $20^{\circ}$ rotation, it may be that a significant number of dots lie at or near the border of the short-range integration limit, and thus ISI can influence the percept reported.

Further evidence that the perception of rocking motion in the rotating displays used here is mediated by the short- 
range process is presented in the next experiments. Following that, additional evidence will be presented for the hypothesis that the percept exhibits cooperativity.

\section{EXPERIMENTS 2 AND 3}

Braddick (1973, 1974), Pantle and Picciano (1976), and Petersik and Pantle (1979) have shown that the activity of the short-range process can be disrupted by brightly illuminating the ISI between stimulus frames or by presenting stimulus frames dichoptically. At the same time, Pantle and Picciano have shown that long-range AM is not affected by dichoptic presentation or illuminated ISIs. Therefore, these manipulations were made in the present experiments to determine whether rocking motion would disappear with illuminated ISIs and dichoptic viewing. Positive findings would suggest that rocking motion is mediated by the short-range process.

\section{Method}

Stimuli and Apparatus. The stimuli for each experiment consisted of the $10^{\circ}$ and $80^{\circ}$ rotation stimuli used in Experiment 1 . The viewing distance and subsequent sizes of the stimuli were the same as in Experiment 1. In Experiment 2, on half of the trials, ISIs were illuminated to match the luminance of the white backgrounds of the stimuli $\left(4.2 \mathrm{~cd} / \mathrm{m}^{2}\right)$. In Experiment 3, the ISI was again dark, but on half of the trials the stimuli were presented dichoptically by means of polarizing filters.

Subjects. The subjects were 20 volunteer psychology majors, 10 of each gender. All reported normal vision, 7 with corrective lenses. Each subject served in both experiments.

Procedure. The subjects were run individually. Each subject was first introduced to examples of rocking and random movement, and this was followed by 10 practice trials with stimuli presented as in Experiment 1 (i.e., binocular viewing with a dark ISI). Next, Experiments 2 and 3 were run in a randomly determined order. Experiment 2 consisted of eight stimulus presentations resulting from the factorial combination of two rotations $\left(10^{\circ}\right.$ and $\left.80^{\circ}\right)$, two ISIs (10 and $80 \mathrm{msec}$ ), and two illumination conditions of the ISI (light vs. dark). Each stimulus presentation consisted of seven cycles of the two-frame stimuli. Trials were run in a random order, and stimulus duration was $200 \mathrm{msec}$. Following each trial, the subject responded either "rocking motion" or "random motion." Experiment 3 consisted of eight stimulus presentations resulting from the factorial combination of the same two rotations and ISIs, along with two viewing conditions: binocular versus dichoptic. Again, trials were run in a random order and stimulus duration was 200 msec. The subject's task was the same as in Experiment 2.

\section{Results and Discussion}

The results for Experiment 2 are shown in Table 1. As can be seen, illumination of the ISI nearly eliminated reports of rocking motion. It was observed that the elimination of rocking motion required some time to develop (perhaps a light-adaptation process was at work); at the 10 -msec ISI, this was about five cycles of the presentation. After rocking motion ceased under illumination conditions, random motion dominated.

The results of Experiment 3 are shown in Table 2. The establishment of motion judgments proved to be difficult with dichoptic viewing, and on many trials subjects reported that they saw no movement or movement that
Table 1

Frequency of Reports of Short-Range (Rocking) Motion in Experiment $2(n=20)$

\begin{tabular}{cccccc}
\hline & \multicolumn{4}{c}{ Viewing Condition } \\
\cline { 2 - 3 } \cline { 5 - 6 } Rotation & \multicolumn{2}{c}{ Dark ISI } & & \multicolumn{2}{c}{ Light ISI } \\
\cline { 2 - 3 } \cline { 5 - 6 } & $10 \mathrm{msec}$ & $80 \mathrm{msec}$ & & $10 \mathrm{msec}$ & $80 \mathrm{msec}$ \\
\hline $80^{\circ}$ & 17 & 16 & & 4 & 3 \\
\hline
\end{tabular}

Table 2

Frequency of Reports of Short-Range (Rocking) Motion in Experiment $3(n=20)$

\begin{tabular}{cccccc} 
& \multicolumn{4}{c}{ Viewing Condition } \\
\cline { 2 - 3 } \cline { 5 - 6 } Rotation & \multicolumn{2}{c}{ Binocular } & & \multicolumn{2}{c}{ Dichoptic } \\
\cline { 2 - 3 } \cline { 5 - 6 } $10^{\circ}$ & $18-m s e c$ ISI & $80-$-msec ISI & & 10 -msec ISI & 80 -msec ISI \\
$80^{\circ}$ & 3 & 16 & & 2 & 3 \\
\hline
\end{tabular}

was neither rocking nor random. Thus, rather than repeat many trials in which a motion judgment could not be made, a third response category was added, "other."3 As Table 2 shows, dichoptic viewing reduced the number of rocking-motion responses, particularly at the 10 -msec ISI. However, random motion was only reported on about $50 \%$ of these trials.

Because an illuminated ISI and dichoptic viewing produced rather dramatic declines in the reports of rocking movement, and because these manipulations have previously been shown to reduce reports of short-range movement, the results of these experiments further support the argument that rocking motion is produced by the short-range process in AM. It should be noted that at least some rocking motion was reported in all conditions of these experiments (see Tables 1 and 2). It is not clear whether such reports of short-range movement under suboptimal conditions represent incomplete elimination of the short-range percept or response bias. In any case, it is not uncommon in studies of AM for subjects to report short-range percepts under conditions that favor the longrange process, and vice versa (e.g., Pantle \& Picciano, 1976; Petersik \& Pantle, 1979). The conclusions offered here are based on the relatively large shifts in the probability of reporting short-range movement over the experimental conditions. Nonetheless, the existence of reports of rocking motion in all conditions requires that the data be interpreted with caution. The final studies in this series provide additional support for the argument that rocking motion also exhibits global cooperativity.

\section{EXPERIMENT 4}

On the basis of the results of the first three experiments, the suggestion was made that the rocking motion perceived in the present displays is based upon the short-range process in AM and that the percept exhibits global cooperativity. Experiment 4 and the subsequent control study 
were designed to further test the hypothesis of global cooperativity. It was reasoned that since dot elements closer to the center of the imaginary circle have smaller linear separations between frames than do those closer to the circumference, they contribute more to the rocking percept (by cooperative means) than do those further from the center. Therefore, placing a mask over dots near the center of the stimulus frames should reduce or eliminate reports of rocking motion, depending upon the size of the mask, the degree of rotation between frames, and the ISI. On the other hand, placing an annulus over dots near the circumference of the invisible circle should, if anything, increase reports of rocking motion (by removing competing "random" signals).

\section{Method}

Stimuli and Apparatus. The apparatus was the same as used in the previous experiments. The stimuli were the same as used previously, with the following modifications. The number of rotations was reduced to four $-10^{\circ}, 20^{\circ}, 40^{\circ}$, and $80^{\circ}$. Three sets of stimuli were subsequently prepared, two with center masks of different sizes and one vith an outer annulus mask. The first set of stimuli (small mask) had a white circle pasted over the center of the stimulus dots. When viewed, this circle had a diameter of $1.4^{\circ}$. The black fixation point was redrawn on the center of the mask to maintain a fixation point. The second set of stimuli (large mask) was similarly prepared, but with a mask having a diameter of $2.9^{\circ}$. The third set had an outer annulus whose width subtended $0.96^{\circ}$. This left visible a center area of dots having a diameter of $2.28^{\circ}$.

Thus, when viewed, the stimuli with the small mask had an outer area in which approximately 27 dots were visible. The large mask allowed approximately 12 dots to be visible. The annulus permitted approximately 22 central dots to be visible.

Subjects. Two groups of subjects were run in two different phases of the experiment. Thirty subjects served in the first phase, in which the masked stimuli were used. Twenty subjects served in the second phase, in which unmasked stimuli were run to obtain baseline data. All subjects were volunteer psychology students, reporting normal vision. There were approximately equal numbers of males and females.

Procedure. Each of the first $\mathbf{3 0}$ subjects was given an explanation of rocking and random movement as in the previous experiments, again followed by 10 practice trials. Next, the subject was randomly exposed to 24 stimulus trials formed by the factorial combination of four rotation angles, two ISIs (10 and $80 \mathrm{msec}$ ), and the three mask conditions. As previously, the ISI was dark and viewing was binocular. Stimulus duration was $150 \mathrm{msec}$. Again following each trial, the subject was to respond that either "rocking" or "random" motion had been perceived.

The second phase of the experiment consisted of the collection of control data from 20 additional subjects. The procedure and viewing conditions were the same as in the first phase, except that no masks were present in the stimuli.

\section{Results}

The results of both phases of the experiment are shown in Figure 4, where the data are plotted separately for the 10-msec ISI (Panel a) and the 80-msec ISI (Panel b) as a function of rotation angle. Separate curves are shown for each mask condition. The first finding of note is that the data from the control conditions correspond well with the data from Experiment 1 (Figure 2), with the following exceptions: for the $10-\mathrm{msec}, 80^{\circ}$ condition, there were (a)

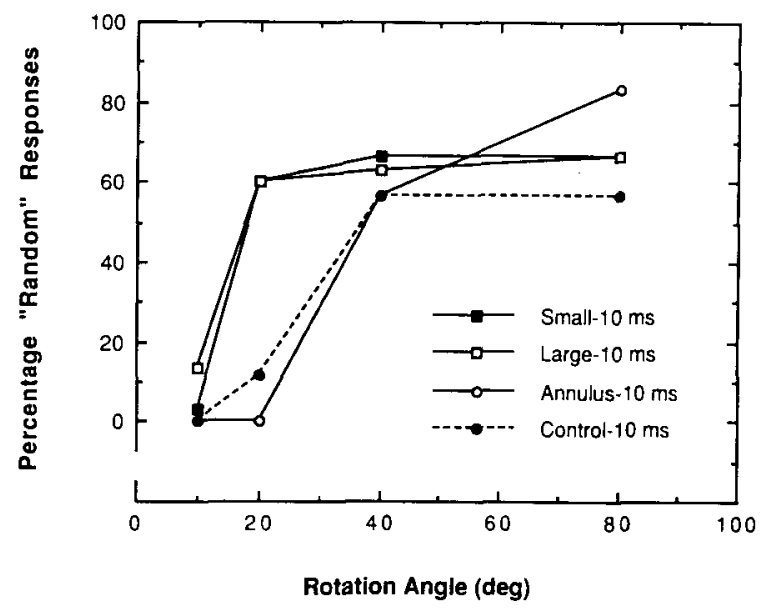

(b)

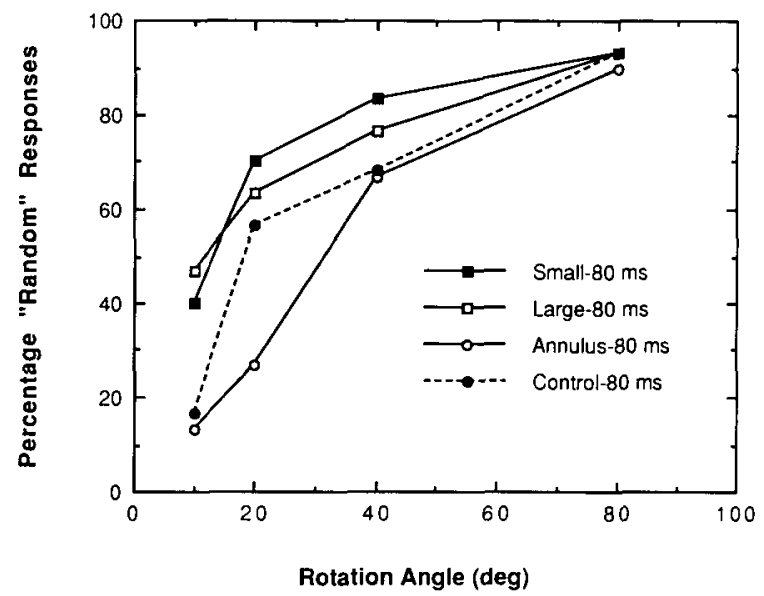

Figure 4. Results of Experiment 4, with the percentage of "random" responses shown as a function of the degree of rotation between corresponding frames of the AM display. Separate curves show the results obtained with central masks of different sizes, an outer annulus, and unmasked (control) stimuli. (a) Results obtained with a 10-msec ISI. (b) Results obtained with an 80-msec ISI.

fewer reports of random movement in the present experiment; for the $80-\mathrm{msec}, 10^{\circ}$ condition, there were somewhat more reports of random movement in the present experiment. These differences could be due to the difference in procedure in the two experiments. Under any circumstance, the trends shown in the data are very similar for the two experiments.

Examining the data for the 10 -msec ISI shown in Figure $4 a$, it can be seen that there was no difference in the reports of random movement between the small and large masks. However, the distributions of response frequencies upon which these data are based were shown to be significantly different for the average of the two mask conditions and the control condition $\left[\chi^{2}(3)=7.43\right.$, $p<.05]$. On the other hand, the control data were not distributed differently from the data obtained with the 
outer annulus $\left[\chi^{2}(3)=2.10, p>.05\right]$. To evaluate differences between mask and annulus conditions (and in view of the fact that the data are from related samples), the McNemar test (Siegel \& Castellan, 1988) was applied to the frequencies of random and rocking responses for each condition at each rotation. Since there were no significant differences between the large- and small-annulus conditions, the present statistical tests were conducted on data obtained with the large-annulus stimuli only. In the overall analysis, this seemed to provide the most conservative test of the null hypothesis. When expected frequencies were small, the binomial test was applied. Frequencies between mask and annulus conditions were significantly different for data obtained with the $20^{\circ}$ rotation stimuli $\left[\chi^{2}(1)=16.06, p<.001\right.$, McNemar test $]$ and with the $80^{\circ}$ stimuli $(p=.035$, binomial test). The frequency difference with the $10^{\circ}$ stimuli approached significance ( $p=.062$, binomial test) and with the $40^{\circ}$ stimuli, failed to reach significance $(p=.50$, binomial test).

Chi-square tests were also conducted on the frequencies that correspond to the percentages shown in Figure 4b. Again, it was found that although the data were in the predicted direction, the data for the control condition were not distributed differently from the data in the annulus condition $\left[\chi^{2}(3)=3.34\right.$ and 2.74 , respectively, both $p s<.05$ ]. Again, McNemar and binomial tests were applied to the frequencies obtained with each level of rotation for the 80 -msec ISI (large-annulus stimuli only). Frequency differences were significant for the $10^{\circ}$ stimuli $\left[\chi^{2}(1)=8.64, p<.01, \mathrm{McNemar}\right.$ test $]$ and for the $20^{\circ}$ stimuli $\left[\chi^{2}(1)=8.64, p<.01\right.$, McNemar test $]$. Differences failed to reach significance for the $40^{\circ}$ and $80^{\circ}$ stimuli $(p=.377$ and .172 , respectively, both binomial tests).

On the basis of these data, it can be concluded that there is evidence that masking the central dots in the apparentrotation display decreases the frequency of rocking-motion percepts relative to the absence of such masking. At the same time, this experiment was not able to discriminate effects due to the sizes of the masks. Also, there was some trend in the data to suggest that an outer annulus increases the frequency of reports of rocking movement; however, this effect was not significant. Thus, it seems likely that placing a mask over dots that have the least linear separation from frame to frame reduces the strength of the short-range motion percept (compared to a no-mask condition). This, in turn, could be due to an elimination or reduction of cooperativity normally produced by those stimulus elements.

\section{CONTROL STUDY}

The argument could be made that the decline in reports of rocking movement found with the central masks used in Experiment 4 could be due to a decrease in the number of elements available with which to solve the correspondence problem. This argument does not seem ten- able because the use of an outer annulus in Experiment 4 did not reduce the number of reports of rocking movement, and even increased them somewhat with the 80-msec ISI. Nonetheless, as a control, a new set of stimuli was made in which there were only 12 dots (corresponding to the approximate number of dots visible with the large mask of Experiment 4). These stimuli were prepared for rotations of $20^{\circ}$ and $40^{\circ}$. Three experienced observers subsequently viewed the new stimuli, as well as the original unmasked stimuli of Experiment 1 (for $10^{\circ}$ and $20^{\circ}$ rotations), at two ISIs, 10 and $40 \mathrm{msec}$. Twenty observations were made for each stimulus set at each rotation and ISI. The frequency of reports of rocking motion for each condition for each subject are shown in Table 3. As can be seen, the decrease in dot density led to more frequent reports of rocking motion (perhaps by eliminating the number of possible false matches), contrary to predictions based on an "absolute number" argument. Furthermore, the 3 subjects observed no obvious differences in the quality of AM with the two sets of stimuli. Therefore, it seems unlikely that the decline in the absolute number of stimulus elements accounts for the decline in reports of rocking movement in Experiment 4 (but see General Discussion).

\section{GENERAL DISCUSSION}

The present experiments present evidence supporting the following conclusions. (1) The "rocking" motion observed in the rotary-motion displays is mediated by the same short-range process previously described with respect to bistable, contour-containing displays (e.g., Braddick \& Adlard, 1978; Gerbino, 1984; Pantle \& Picciano, 1976; Petersik \& Pantle, 1979). This conclusion is supported by the fact that rocking motion is dominant at short ISIs and small separations between corresponding elements across frames (Experiment 1), and by the fact that rocking motion is disrupted by illuminated ISIs and dichoptic viewing (Experiments 2 and 3). (2) The rocking motion observed in the present displays exhibits global cooperativity. This is supported by the observation that rocking motion is perceived at the perimeter of

Table 3

Percentage of Reports of Short-Range (Rocking) Motion in the Control Study (3 subjects, 20 trials per condition)

\begin{tabular}{|c|c|c|c|c|}
\hline \multirow[b]{2}{*}{ Rotation } & \multicolumn{2}{|c|}{ 12-dot Stimuli } & \multicolumn{2}{|c|}{ 40-dot Stimuli } \\
\hline & 10-msec ISI & 40-msec ISI & 10-msec ISI & 40-msec ISI \\
\hline \multicolumn{5}{|c|}{ Subject J.T.P. } \\
\hline $20^{\circ}$ & 100 & 100 & 100 & 90 \\
\hline $40^{\circ}$ & 25 & 40 & 10 & 0 \\
\hline \multicolumn{5}{|c|}{ Subject A.L.R. } \\
\hline $20^{\circ}$ & 100 & 100 & 50 & 45 \\
\hline $40^{\circ}$ & 100 & 100 & 0 & 0 \\
\hline \multicolumn{5}{|c|}{ Subject K.E.V. } \\
\hline $20^{\circ}$ & 100 & 100 & 85 & 70 \\
\hline $40^{\circ}$ & 95 & 95 & 20 & 20 \\
\hline
\end{tabular}


the circular stimulus areas (and, indeed, throughout the entire stimulus area), even when corresponding stimulus elements across frames are separated by distances outside the presumed spatial integration limit of the shortrange process (Experiment 1 ). In addition, masking areas in the center of these circular displays (areas in which stimulus-element separations are within the short-range integration limit) decreases reports of rocking motion (Experiment 4). (3) By inference, on the basis of the first two conclusions, and because certain similarities between the short-range process observed in $\mathrm{r}-\mathrm{d}$ displays and contourcontaining displays have already been reported (e.g., Petersik, 1989; Petersik \& Pantle, 1979), it can be concluded that the same short-range process mediates form perception in alternating $\mathrm{r}-\mathrm{d}$ displays and movement (or stationarity) perception in certain bistable contourcontaining displays.

An alternative interpretation of the present experiments is based upon "correspondence matching" (Ullman, 1979,1980 ). That is, AM displays containing multiple elements present a situation in which the visual system must choose, for each element in the first frame, a corresponding partner in the second frame to which $A M$ is imparted. Among the "guidelines"' Ullman (1979) identified with the correspondence process are an affinity measure partially based upon the distance between elements in successive frames: all other influences being equal, correspondence matches across frames should be between nearest neighbors. In terms of the present experiments, the greater the rotation of stimulus elements between frames, the greater should be the likelihood of false matches, that is, the greater will be the chance that the second-frame nearest neighbor of any first-frame dot will be different from that dot's own rotated position in Frame 2. Since such matches should be random (i.e., based upon the original random positioning of the dots), the resulting AM should also give the appearance of randomness. Thus, the increase in reports of random movement with increases in angle of rotation could reflect an increase in false correspondence matches rather than the breakdown of the short-range process. This interpretation is supported by the fact that in the control study, a reduction in the density of stimulus dots, which would also decrease the number of potential false correspondence matches, resulted in a decrease in the relative number of random-movement reports.

The position taken here is that correspondence matching cannot be studied independently of the perceptual mechanisms that underlie the hypothetical short- and longrange processes. For example, correspondence matches are strongly influenced by adaptation of short-range or long-range percepts, or by manipulations involving illuminated ISIs or dichoptic viewing (all three are effective over a wide range of ISIs; Petersik \& Pantle, 1979). Indeed, the fact that, in the present experiments, percepts of random movement were increased with illuminated ISIs and with dichoptic viewing suggests a role for the shortrange process. What cannot be logically determined from the present data is whether the facilitation of random movement with increasing angles of rotation reflects an inherent limitation of the short-range process or an enhanced contribution of false correspondence matches. Probably the two are not independent: AM percepts are strongly influenced by constraints on possible correspondence matches (e.g., Ullman, 1980), and correspondence matches are influenced by the nature of the AM process favored by such parameters as ISI, stimulus duration, and viewing conditions. The latter notion is evidenced by the fact that correspondence matches change drastically in both $\mathrm{r}-\mathrm{d}$ displays (leading from percepts of the coherent movement of a correlated target area under the influence of the short-range process to percepts of incoherent movement when the short-range process is not favored) and contour-containing displays (e.g., the change from endto-end movement to group movement in the Termus display) as ISI progresses from short to long. Therefore, any study of correspondence matching must simultaneously consider the AM process favored by the displays. Since the present experiments were designed to test AM percepts over conditions both favorable and unfavorable to the short-range process, the effect of angle of rotation can probably best be interpreted as reflecting a dominance of the nearest-neighbor principle of correspondence matching under conditions that do not favor the global cooperativity (and consequent rocking movement) otherwise produced by the short-range process.

The above discussion has offered a correspondencematching explanation of random movement. In keeping with the emphasis on interpretations that also consider AM processes, I now consider a process-based explanation of random movement. Since the present experiments were primarily designed to examine the role of the short-range process in rocking movement, and since no experimental tests of hypotheses relating to random motion have been conducted, the following arguments are based solely upon observation. Some AM displays that contain global contours yield alternative motion percepts. For example, the Ternus display used by Braddick and Adlard (1978), Pantle and Picciano (1976), and Petersik and Pantle (1979) yields percepts of "end-to-end movement" and "group movement." The former is considered to be mediated by the short-range process, whereas the latter is thought to be mediated by the long-range process in AM (cf. Braddick, 1974). " "Random motion" does not seem to be solely mediated by the long-range percept for the following reasons. First, there is no common motion vector in which all elements partake, as is seen in, for example, group movement (Pantle \& Picciano, 1976), where the elements move in toto. Second, stimulus matches over frames seem to be random and chaotic: some dots move over short differences, some over long. Furthermore, some dots seem to change their motion paths over different cycles of a display. This chaotic motion seems also to be inconsistent with the coherent motion that is often produced with the globally cooperative short-range process (e.g., rocking motion or the establishment of 
figures in alternating $\mathrm{r}-\mathrm{d}$ displays). Since global cooperativity is thought to be established by the spatial pooling of short-range activity (Chang \& Julesz, 1985), the suggestion is made here that "random motion" is the product of the independent activity of the long-range process and unpooled short-range activity. This would account for both the chaotic appearance and the range of distances over which motion is perceived in what has been called random motion, and is consistent with the previous observation that short-range and long-range percepts can coexist in the same AM display (Braddick \& Adlard, 1978).

Although there is no coherent overall theoretical structure that integrates notions of short-range and long-range processing, there is a sizable body of literature that supports the two-process distinction (Petersik, 1989). Indeed, the present experiments dovetail nicely with the "heuristic theoretical perspective" offered by Petersik (1989), which suggests that the short-range process can generate globally cooperative percepts through the spatial pooling of hypothetical "short-range units."

\section{REFERENCES}

BELL, H. H., \& LAPPIN, J. S. (1979). The detection of rotation in randomdot patterns. Perception \& Psychophysics, 26, 415-417.

Bischof, W. F., Groner, M. (1985). Beyond the displacement limit: An analysis of short-range processes in apparent motion. Vision Ressearch, 25, 839-848.

BradDick, O. J. (1973). The masking of apparent motion in randomdot patterns. Vision Research, 13, 355-369.

BrADDICK, O. J. (1974). A short-range process in apparent motion. Vision Research, 14, 519-527.

Braddick, O. J., AdLARD, A. J. (1978). Apparent motion and the motion detector. In J. Armington, J. Krauskopt, \& B. R. Wooten (Eds.), Visual psychophysics and physiology (pp. 417-426). New York: Academic Press.

Chang, J. J., \& Julesz, B. (1984). Cooperative phenomena in apparent movement perception of random-dot cinematograms. Vision Research, 24, 1781-1788.

Chang, J. J., \&ulesz, B. (1985). Cooperative and non-cooperative processes of apparent movement of random-dot cinematograms. Spatial Vision, 1, 39-45.

Gerbino, W. (1984). Low-level and high-level processes in the perceptual organization of three-dimensional apparent motion. Perception, 13, 417-428.

Julesz, B. (1971). Foundations of Cyclopean perception. Chicago: University of Chicago Press.

Pantle, A. J., \& Petersik, J. T. (1980). Effects of spatial parameters on the perceptual organization of a bistable motion display. Perception \& Psychophysics, 27, 307-312.

Pantle, A. J., \& Picciano, L. (1976). A multistable movement display: Evidence for two separate motion systems in human vision. Science, 193, 500-502.
Petersik, J. T. (1989). The two-process distinction in apparent motion. Psychological Bulletin, 106, 107-127.

Petersik, J. T., \& Pantle, A. J. (1979). Factors controlling the competing sensations produced by a bistable stroboscopic motion display. Vision Research, 19, 143-154.

Petersik, J. T., Pufahl, R., Krasnoff, E. (1983). Failure to find an absolute retinal limit of a putative short-range process in apparent motion. Vision Research, 23, 1663-1670.

Siegel, S., CASTELlaN, J. N., JR. (1988). Nonparametric statistics for the behavioral sciences. New York: McGraw-Hill.

Ullman, S. (1979). The interpretation of visual motion. Cambridge, MA: MIT Press.

Uliman, S. (1980). The effect of similarity between line segments on the correspondence strength in apparent motion. Perception, 9 , 617-626.

\section{NOTES}

1. These frames differ from classical $r-d$ frames in two ways. First, the stimulus elements of the present displays are outline contours rather than filled pixels. Second, and more importantly, these contours are randomly scattered on a homogeneous white background. In $r-d$ frames, every pixel is a potential stimulus having a potential partner in the alternate frame. Therefore, in $r-d$ frames, it is meaningless to think of figures and backgrounds in the frames, at least prior to their alternation when a form may emerge due to the activity of the short-range process. In the present frames, the forms are clearly defined, as is the background, and they are visible prior to alternation.

2. The response criteria were designed to reduce the likelihood of falsely identifying conditions that yield a global percept. The first 5 subjects were questioned regarding their ability to use the above criteria: they reported that the task was generally easy and that percepts with obvious nonrocking motion had been called "random." Four of the 5 subjects also reported instances of being unsure how to respond, primarily because during the brief period of presentation "one or two" contours "may have" made random motions in otherwise rocking percepts. In such instances, and for the remaining 13 subjects, the subjects were instructed to use their best judgment regarding how to name the percept. In most cases, such displays were subsequently identified as rocking. My best impression is that these instances accounted for less than $10 \%$ of all trials.

3. This conclusion was reached after the first 2 subjects found it difficult to categorize some dichoptic motion displays. Following this, the experiment was modified to include the "other" category, and 2 new subjects were run to replace the initial 2 .

4. Characteristics of the long-range process are commonly thought to include a large spatial range over which matches are made (up to several degrees); a preference for relatively long ISIs (usually greater than $80 \mathrm{msec}$ ); a preference for longer stimulus durations than the shortrange process; a lack of sensitivity to stimulus contrast, illuminated ISIs, or dissimilarity of forms; and an attentive, serial processing mode (see Petersik, 1989, for a review).

(Manuscript received May 25, 1989; revision accepted for publication October 16, 1989.) 